\title{
ASSISTÊNCIA DE ENFERMAGEM A PACIENTE COM FIBRILAÇÃO ATRIAL
}

\author{
Nursing assistance to patients with atrial fibrillation \\ Asistencia de enfermería al paciente con fibrilación atrial
}

Lutgarde Magda Suzanne Vanheusden

Deyse Conceição Santoro

\section{Resumo}

Este estudo tem por objetivo apresentar uma revisão atualizada sobre as questões que envolvem a assistência à paciente que desenvolve fibrilação atrial. Essa revisão discute a condição complexa dessa taquiarritmia que influencia a mortalidade, morbidade, abordando o seu elevado custo para o sistema de saúde. A assistência e o importante papel da educação para a enfermagem nessa área estão sendo discutidos. Como a prevalência da fibrilação atrial aumenta com a idade e 0 B rasil tem uma população idosa cada vez mais crescente, a enfermagem enfrenta atualmente 0 desafio para cuidar dessa população que apresenta necessidades variadas.

Palavras-chave: Enfermagem. Fibrilação atrial. Arritmia.

\begin{abstract}
The objective of this study is to present an up to date revision about the assistance of the patient that develops atrial fibrilation. This revision raises the discussion on the complexity of atrial fibrillation that influences the mortality, morbidity, and the high cost for the health system. The assistance and the important paper of education of nursing on atrial fibrillation are here discussed. The population of Brazil is increasing in time and age consequently the prevalence of atrial fibrillation is also increasing. Thus, nursing faces the current challenge to take care of this specific population that presents varied necessities.
\end{abstract}

\section{Resumen}

Este estudio tiene por objetivo presentar una revisión actualizada sobre las cuestiones que envuelven la asistencia al paciente que desarrolla fibrilación atrial. Esa revisión discute la condición compleja de esa taquiarritmia que influencia la morbidad, mortalidad, enfocando el costo que es muy grande para el sistema de salud. Se discute también la asistencia y el impor tante papel de la educación para la enfermería en esa área. Como la prevalencia de la fibrilación atrial aumenta con la edad y como el Brasil tiene una población de ancianos cada vez más creciente, la enfermería, actualmente, enfrenta el desafío para cuidar de esa población que presenta necesidades variadas.
Keywords:

Nursing. Atrial fibrillation. Arrhythmia.
Palabras clave:

Enfermería. Fibrilación atrial. Arritmia. 


\section{INTRODUÇÃO}

Fibrilação atrial (FA) é uma condição complexa que influencia a mortalidade, morbidade, qualidade de vida do indivíduo e que impõe um gasto financeiro muito grande para o sistema de saúde. A expectativa é que 0 número de pessoas com fibrilação atrial aumente progressivamente, pois o risco de sua ocorrência elevase com o crescimento da idade. Desde a última década, a fibrilação atrial é objeto de investigação clínica e epidemiológica. Como resultado dessas investigações, novos dados são disponibilizados e opções inovadoras de tratamento são sugeridas.

A fibrilação atrial é a arritmia sustentada mais prevalente e responsável pelo maior número de internações e com uma duração maior que qualquer outra arritmia ${ }^{1}$. 0 paciente precisa na maioria das vezes de hospitalizações repetidas em função do caratér recorrente da fibrilação atrial. 0 custo por causa desta arritmia é consideravel devido ao número de indivíduos que apresentam esta condição mórbida pela sua taxa de recorrência e conseqüente re-hospitalização.

Um estudo sobre a distribuição de custo de cuidados a pacientes com fibrilação atrial mostrou que 0 principal custo desse paciente é a hospitalização $(52 \%)$, seguido por gasto com drogas $(23 \%)$, consultas $(9 \%)$, investigações diagnosticas (8\%), perda de trabalho $(6 \%)$ e procedimentos paramédicos $(2 \%)$. As principais causas de internação desses pacientes são a admissão hospitalar para cardioversão, seguida por insuficiencia cardíaca e implante de marcapasso. Adicionalmente algumas internações visavam iniciar a terapia antiarritmica. 0 autor recomenda a organização de clínicos especializados em fibrilação atrial com um grupo interdisciplinar de enfermagem, nutricionista, psicólogos para reduzir os custos².

A fibrilação atrial não é uma arritmia letal, mas está associada com mortalitade e morbidade aumentadas. Pacientes com fibrilação atrial têm duas vezes mais 0 risco de óbito, e sem uma terapia antitrombólica efetiva existe um risco cinco vezes maior de occorência de um acidente vascular encefálico ou embolia pulmonar. A fribrilação atrial com frequência ventricular alta apresenta um risco de induzir cardiomiopatia (taquicardiomiopatia) ${ }^{3}$. Devido à frequência ventricular aumentada e a perda de "kick" atrial, a insuficiência cardíaca e a isquemia existente podem piorar.

Como o número de pessoas com fibrilação atrial está aumentando e existem várias opções de tratamento, enfermeiros em várias unidades (como emergência, unidade coronariana, ambulatorial, de eletrofisiologia e unidade de internação) atendem essa população com as mais variadas necessidades.

\section{DESCRIÇÃO}

A fibrilação atrial é uma taquiarritmia supraventricular caracterizada por uma ativação atrial incoordenada. A ativação caótica do átrio tem como consequência uma perda no enchimento do ventrículo, o "kick" atrial, que pode ser responsável por uma perda substancial de até $30 \%$. Ao electrocardiograma as ondas $P$ são substituídas por ondas fibrilatórias, as ondas $f$, que variam em aplitude, forma e duração. Associado a isto, temos uma resposta ventricular irregular e freqüentemente rápida.

A combinação da perda do "kick" atrial, resposta ventricular rápida e irregular prejudica o enchimento ventricular e pode resultar em uma redução substancial do débito cardíaco, e o paciente pode ter sintomas como palpitações, dispnéia, tontura, cansaço e dor no peito. A ocorência de fibrilação atrial pode variar individualmente, alguns têm episódios paroxísticos infrequentes, outros têm fibrilação atrial permanente.

A fibrilação atrial pode ocorrer de forma transitória, acompanhando situações clínicas específicas (infarto do miocárdio, doença pulmonar, medicamentos), ou apresentar-se sob a forma crônica, recorrente ou incessante. Em 30\%, a FA ocorre na ausência de cardiopatia estrutural (FA solitária). A presença de uma fibrilação permanente com a freqüência cardíaca média elevada (>100 bpm em uma monitorização eletrocardiográfica ambulatorial de $24 \mathrm{~h}$ ) sem controle farmacológico da resposta ventricular, evolui quase sempre para disfunção ventricular, configurando 0 quadro clínico de taquicardiomiopatia ${ }^{3}$.

A classificação da fibrilação atrial segundo as Diretrizes da Sociedade Brasileira de Cardiologia4, incluiu as seguintes definições:

FA inicial - primeira detecção, sintomática ou não da arritmia, desde que a duração não seja superior a $30 \mathrm{~s}$;

FA crônica - a documentação da recorrência da arritmia pode apresentar três formas distintas:

1. Paroxística - episódios com duração de até 7 dias; os surtos são geralmente autolimitados e revertem espontaneamente a ritmo sinusal;

2. Persistente - episódios com duração superior a 7 dias; este limite é arbitrário e define um período em que a reversão espontânea é pouco provável e a reversão farmacológica raramente ocorre, geralmente necessitando cardioversão; pode ser a primeira apresentação clínica da FA ou ser precedida por crises recorrentes; 
3. Permanente - a FA é documentada já há algum tempo e a reversão a ritmo sinusal, farmacológica ou elétrica é ineficaz.

\section{TRATAMENTO FARMACOLÓGICO E NÃO FARMACOLÓGICO}

0 conhecimento e as novas tecnologias abriram 0 caminho para as mais variadas opções de tratamento da fibrilação atrial. 0 tratamento do fibrilação atrial pode ser divido em três grandes objetivos. 0 primeiro é o reestabelicimento do ritmo sinusal e manutenção desse ritmo. 0 segundo é o controle da frequência e o terceiro é a redução das complicações tromboembólicas. Para atingir esses objetivos, 0 tratamento da fibrilação atrial pode ser farmacológico ou não farmacológico.

\section{Tratamento farmacológico}

A terapia farmacológica é geralmente a terapia inicial para pacientes com fibrilação atrial. Várias drogas antiarrítmicas estão disponíveis no mercado para reestabelecer ritmo sinusal, controlar a frequência cardíaca ou diminuir 0 risco de fenomênos tromboembólicos.

Recente estudo do AFFIRM ${ }^{5}$ sugere que o controle da frequência pode ser a terapia de escolha para pacientes com fibrilação atrial com faixa etária elevada. Para reduzir o risco de taquicardiomiopatia, a medicação deveria estar ajustada para controlar a frequência cardíaca entre 60-80 bpm em repouso e 90-115 bpm em atividade.

A reversão para ritmo sinusal com drogas é mais eficaz na FA inicial com duração menor que 7 dias. A reversão farmacológica para ritmo sinusal é mais simples que a reversão com cardioversão não necessitando de anestesia geral. A reversão farmacológica pode ser feita com paciente internado ou não. Quando o paciente tem uma doença cardíaca associada ou outro distúrbio de condução interventricular, eles são geralmente internados para monitorar a terapia antiarrítmica, visando prevenir a sua deleteriação pro-arrítmica.

Pacientes com fibrilação atrial têm um risco aumentado para eventos tromboembólicos devido à lentificação do fluxo sanguíneo nos átrios que induz a formação de trombos. 0 acidente vascular encefálico é uma complicação severa da fibrilação atrial e o uso de anticoagulantes reduz esse risco. 0 uso de anticoagulantes é geralmente indicado para pacientes com fibrilação atrial, independente da fibrialação atrial ser paroxística, persistente ou permanente. A indicação de warfarina como droga anticoagulante para os pacientes com alto e moderado risco para acidente vascular encefálico ${ }^{6}$, por exemplo, requer dose periodicamente ajustada e controlada pelo exame de sangue Tempo de Protrombina (TP), que pode ser responsabilidade do enfermeiro. 0 valor do tempo de protrobina é referido com o INR (International Normalized Rate) que é uma taxa universal e tem que estar entre 2,0-3,0. Sem uso de anticoagulante, 0 valor normal é de 1,0. No caso de pacientes com um baixo risco de acidente vascular encefálico, 0 tratamento profilático pode ser feito com aspirina $325 \mathrm{mg}$ por dia, com o qual a preocupação e o controle passam a ser focados na irritação da mucosa gástrica.

\section{Terapia não farmacológica}

A terapia não farmacológica para fibrilação atrial está em ritmo crescente. 0 tratamento com medicação pode ter efeitos colaterais e pode aumentar o risco para eventos pró-arritmicos e conseqüente risco de morte súbita. As medicações podem ter um efeito limitado de sucesso e um custo mais alto que a terapia farmacológica. A terapia não farmacológica é uma opção que tem despertado bastante interesse. No momento, existe a opção de cardioversão elétrica, ablação por radiofreqüência, estimulação atrial profilática e cirurgia.

\section{a. Cardioversão elétrica}

A cardioversão elétrica é a opção mais efetiva para reestabelecer 0 ritmo sinusal. Com a cardioversão, as células atriais são depolarizadas e o nó sinusal pode estimular os átrios novamente. Para que a cardioversão elétrica seja realizada, o paciente tem que estar internado e isto eleva o custo do tratamento da fibrilação atrial e interfere no cotidiano do paciente e sua familia.

A cardioversão elétrica geralmente tem poucos riscos e sua taxa de sucesso de 70 a $90 \%$, mas precisa ser realizada sob sedação e o paciente deve estar em jejum. ${ }^{7}$ 0 choque deve ser sincronisado com 0 QRS para evitar a indução de fibrilação ventricular. A energia inicial para reversão de fibrilação atrial é de $200 \mathrm{~J}$. Nos casos em que a fibrilação atrial não é revertida para ritmo sinusal, aumenta-se a energia em 100 J até chegar a 360 J. Utilizando-se novos cardioversores bi-fásicos, pode-se iniciar a cardioversão com 100 J elevando-se para 150 e $200 \mathrm{~J}$ em casa de insucesso. A atuação do enfermeiro no momento dessas programações e acompanhamento da resposta do paciente ao procedimento é essencial ${ }^{8}$. 
0 cardioversão elétrica pode ser influenciado pelo posicionamento das pás-eletrodos, cardioversor monofásico ou bifásico e administração de drogas antiarrítmicas antes do procedimento. A administração das drogas anti-arrítmicas também ajuda a prevenir a recorrência da fibrilação atrial pós-cardioversão elétrica. É comum o paciente sentir-se inseguro e necessitar de apoio e esclarecimentos sobre o procedimento.

Em caso de cardioversão elétrica ou farmacológica, o paciente é anticoagulado por 3 semanas antes da cardioversão, caso a fibrilação atrial já esteja presente há mais de 48 horas, pois neste caso o risco da existência de trombos é maior. Após a cardioversão, o paciente deve permanecer anticoagulado durante 3 a 4 semanas, até 0 pleno restabelecimento da função contrátil do átrio.

As complicações possíveis na cardioversão elétrica são: irritação da pele, queimadura local, bradicardia, taquicardia e fibrilação ventricular, tromboembolia arterial e complicações relacionadas à anestesia. 0 cuidado com o preparo do gel condutor sobre as pás (gel salino) ou compressas umedecidas em solução salina sobre os locais de posicionamento das pás evitam as queimaduras locais e são de responsabilidade do enfermeiro. A monitorização precisa e a observação constante da resposta eletrocardiográfica do paciente após a cardioversão elétrica permite a detecção precoce de outras complicações arrítmicas e intervenção imediata. Além da presença do anestesista, a providência de material e dispositivos ventilatórios para o caso de uma intercorrência respiratória fazem parte dos cuidados dispensados pelo enfermeiro. As repetidas internações e cardioversões às quais o paciente tem que se submeter interferem bastante na sua qualidade de vida.

\section{b. Ablação por radiofrequência}

Ablação por radiofreqüência pode ser feita para controle da frequência ou para reversão a ritmo sinusal. Para controle da freqüência, é feita a ablação do nódulo atrioventricular, provocando-se um bloqueio atrioventricular total intensional e o paciente é submetido a estimulação cardíaca permanente. Os pacientes que fazem este procedimento ficam em fibrilação atrial e precisam de anticoagulação crônica, necessitando da atenção do enfermeiro em tempo integral durante o procedimento ${ }^{9}$.

Recentemente, os estudos demonstraram ser possível a realização da ablação de áreas específicas que podem deflagrar a fibrilação atrial. Foi demonstrada a presença de extensões do tecido miocárdico do átrio esquerdo para as veias pulmonares, especialmente sua porção ostial. Estes prolongamentos musculares favorecem a ocorrência de focos ectópicos rápidos. Outros locais no átrio direito, como a crista terminalis, e porções proximais do óstio do seio coronário e da desembocadura da veia cava inferior e superior podem apresentar focos indutores de arritmias atriais.

A ablação ostial e em volta do ostio das veias pulmonares pode ser bem succedida para reduzir episódios de fibrilação atrial em alguns pacientes. 0 sucesso deste procedimento depende de localizar e fazer ablação de todos os focos ou conexões que podem induzir fibrilação atrial. Este procedimento de ablação pode apresentar complicações como estenose pulmonar, tamponamento cardíaco, tromboembolia, paralesia do nervo frênico, perfuração de aorta e fístula esôfago-átrio esquerdo. A ablação do foco arritmogênico através de radiofreqüência atinge um sucesso terapêutico em 60 a $80 \%$ dos casos ${ }^{9}$. Durante a realização de uma ablação, 0 enfermeiro deve ter à disposição medicamentos e dispositivos necessários para a emergência cardiológica (carrinho para parada cardiopulmonar, marca-passo, antiarrítmicos, entre outros).

As recomendações atuais para ablação de fibrilação atrial, pela Diretriz da Sociedade Brasileira de Cardiologia ${ }^{10}$, são:

Fibrilação atrial deflagrada por outra taquicardia - Ablação dos circuitos primários de pacientes com sindrome de Wolf-Parkinson-White, taquicardia por reentrada nodal, flutter atrial e taquicardia atrial focal (I);

Isolamento das veias pulmonares- 1) FA paroxística assintomática sem cardiopatia estrutural ou disfunção sinusal, com resposta ventricular rápida nãoresponsiva a pelo menos duas drogas antiarrítmicas (II a ); 2) FA paroxística ou persistente sem cardiopatia estrutural, assintomática, de difícil controle clinico, evoluindo com disfunção ventricular esquerda secundária a arritmia (II a) 3) FA persistente ou permanente sintomática sem cardiopatia estrutural ou disfunção sinusal não-responsiva a pelo menos duas drogas antiarrítmicas (II b) 4) FA controlada com drogas antiarrítmicas (III);

Ablação do nó AV e implante de marca-passo definitivo- 1) FA paroxística, persistente ou permanente com resposta ventricular rápida não controlada com tratamento farmacológico e não farmacológico ( I ) 2) em pacientes idosos ou com co-morbidades ( I);

Ablação do circuito do flutter atrial - 1) Flutter atrial comum (relacionado ao istmo veia cava inferior - anel da valva tricúspide) ( 1); 2) Flutter atrial atípico ( não-relacionado ao istmo veia cava inferior-anel da 
valva tricúspide ) Técnica convencional (II b), técnica com mapeamento eletroanatômico ( I );

Ablação do nó AV e implante de marcapasso em pacientes com flutter atrial - 1) Flutter atrial com resposta ventricular rápida ou com baixa débito, não controlado com drogas anti-arrítmicas ou com tratamento não farmacológico ( I ); 2) Flutter atrial controlado com drogas antiarrítmicas ou com possibilidade de ablação curativa da arritmia ( III ).

\section{c. Tratamento cirúrgico}

0 procedimento cirúrgico para a fibrilação atrial pode ser uma opção terapêutica para alguns pacientes. Em 1991, foi desenvolvido um procedimento denominado cirurgia do labirinto (Maze) visando a manutenção do ritmo sinusal e manter a condução atrioventricular e restaurar a contração atrial. A cirurgia de Maze é indicada para pacientes que necessitam de cirurgia cardíaca por outras causas (como a troca de válvula mitral). A técnica consiste em realizar várias incisões cirúrgicas em ambos os átrios para construir um labirinto, tirar os apêndices atriais e isolar as veias pulmonares. Modificações recentes com uso de crioablação ou de radiofreqüência para fazer linhas de bloqueios substituindo as incisões cirúrgicas, encurtaram o tempo cirúrgico diminuindo os riscos. Os cuidados com o paciente são os mesmos para todos aqueles submetidos a uma cirurgia.

\section{O ENFERMEIRO E O PACIENTE COM FIBRILAÇÃO ATRIAL}

Com 0 aumento progressivo dos pacientes com fibrilação atrial e a evolução do conhecimento e do tratamento da fibrilação atrial, o profisional de enfermagem tem cada vez mais tido contato com esse tipo de paciente com necessidades variadas. No cuidado, é importante a interação da enfermeira com o paciente, tomando em consideração as necessidades objetivas e subjetivas dele ${ }^{11}$.

0 paciente com fibrilação atrial pode ser internado para fazer cardioversão elétrica, ser submetido a um procedimeto ablativo, iniciar um tratamento com droga ou internar-se devido a uma outra doença associada, como cardiopatia isquêmica, ou ainda por conta de um acidente vascular encefálico.

Um aspecto importante do cuidado de enfermagem é o suporte educativo para o paciente com fibrilação atrial. Varios tópicos podem ser 0 objeto dessa educação. Um desses tópicos refere-se ao uso de medicamentos para controle da arritmia ou para prevenção de eventos tromboembólicos. 0 paciente precisa ter o conhecimento de que os antiarrítmicos podem ter diversos efeitos colaterais, como hipotensão, tontura, bradicardia, náusea e vômito, insônia, taquicardias, síncope, reação alérgica, dor no peito, tosse, perda de apetite, diarréia, constipação, que precisam ser comunicados para o enfermeiro ou médico assistente. Alguns outros medicamentos podem interferir com a ação dos antiarrítmicos e o paciente sempre deve, antes de iniciar ou interromper qualquer medicação, solicitar orientação. 0 paciente deve saber 0 risco do uso de outros medicamentos quando se encontra em uso de antiarrítmicos. A combinação de dois antiarrítmicos pode piorar as arritmias ou dar origem as outras. É adequado evitar a condução de automóveis até ter-se a noção dos efeitos da medicação. A interação medicamentosa de alguns antiarrítmicos com a medicação anti-coagulante também precisa ser investigada e acompanhada pela equipe clínica (enfermeiro e médico) ${ }^{12}$.

Deve-se orientar os pacientes, de maneira bem clara, lembrando que, durante um episódio de fibrilação atrial, sintomas como palpitacões, cansaço e dispnéia podem ocorrer. Eles devem saber que crises totalmente assintomáticas também podem ocorrer. Em alguns pacientes, a fibrilação atrial pode estar relacionada ao de álcool ou de cafeína. A orientação no sentido de limitar o uso do álcool e da cafeína, presentes no café, chá, coca-cola deve ser feita.

Os sintomas de fadiga e dispnéia podem limitar as atividades sociais e físicas do paciente. Por isso, é de grande ajuda que os pacientes alternem descanso e atividade fisica para manter a energia com manutenção das atividades diárias. É sabido que alguns pacientes podem limitar suas atividades socais com medo de ter episódios de fibrilação atrial, entretanto, os episódios podem estar associados com estresse emocional e mudança na vida diária.

A prevenção de eventos tromboembólicos é rotineiramente feita com 0 uso de anti-coagulantes. 0 paciente deve ser informado sobre a importância do uso dessa medicação de maneira rotineira e da necessidade do seu controle, das interações com certos alimentos e outras drogas. A warfarina, por exemplo, deve ser sempre tomada uma vez por dia e seu uso em jejum, pela manhã, pode aumentar a absorção. Quando o paciente está em período de ajuste da dose, este deve ser feito sempre que o tempo de protrombina se mostra inadequado. Em caso de esquecimento, pode tomar a mesma dose, caso o paciente se lembre no 
mesmo dia. Não pode tomar uma dose dupla para compensar uma dosa esquecida, e nunca pode mudar a dosagem do anticoagulante sem ter se communicado com médico assistente. Nos casos de não usar 0 anticoagulante por alguns dias, os níveis do INR tornamse inadequados e, dessa forma, um exame do tempo de protrombina deve ser avaliado para reiniciar o uso da warfarina e seu ajuste de dose ${ }^{6}$.

A warfarina pode ter interações com outras medicações como aspirina, drogas anti-arritmicas e produtos que contêm vitamina K. O INR deve ser mantido entre 2-3 e deve ser avaliado regularmente para controle do anticoagulante. 0 paciente deve ser informado sobre os sinais de sangramento ou de doenças que podem ocorrer. Os sintomas que podem ocorrer incluem: cansaço, palidez (anemia), cortes que não param de sangrar depois de ter aplicada pressão de dez minutos, sangramento do nariz ou gengiva, sangramento na urina ou fezes, vômito ou tosse acompanhados de sangramento que também podem parecer com borra de café, dor de cabeça incomum, tonteira, dor ou edema incomum, hematomas que aparecem sem razões, dificuldade para respirar.

A dieta deve ser balanceada e equilibrada. Alterar muito a quantidade da comida que tem um teor alto de vitamina $K$ pode modificar a ação da warfarina 6 . Comida que contém alto teor de vitamina K: brocolis, espinafre, laranja, couve, couve-flor, repolho, agrião, aspargo, ervilha, alface, figado, abacate, azeite de oliva, agua de coco. É melhor evitar álcool durante 0 uso de warfarina, pois, como comentado anteriormente, 0 álcool interfere com a ação do anticoagulante.

0 paciente deve saber da importância de informar o seu dentista/cirurgião que ele se encontra em uso de warfarina. É possível que, no caso de algum procedimento, ele tenha que checar o INR e precise parar de tomar warfarina por alguns dias.

A prevenção de acidentes é de importância em pacientes em uso de anticoagulante. Evitar situações onde o paciente pode se machucar em casa ou no trabalho, com esportes radicais ou em grupo. Melhor esporte a ser praticado pelo paciente anticoagulado é o esporte individual. Em caso de corte na pele, é necessária pressão constante no local por 10 minutos. Em caso de doenças com sintomas gastrointestimais como vômitos, diarreia, infecção ou febre, é bom comunicar-se imediatamente com o médico assistente, porque essas ocorrências podem mudar o modo de ação da warfarina ${ }^{6}$. 0 paciente deve ser alertado que em caso de viagens é sempre bom ter a medicação junto a si, não a deixando no carro ou na bagagem.
É normal que pacientes que se submetam a uma cardioversão elétrica ou a uma ablação por radiofreqüência sintam-se inseguros, ansiosos ou amedrontados. 0 enfermeiro pode reduzir a ansiedade e medo do paciente explicando o procedimento e falando sobre as sensações que ele pode sentir durante ou após o procedimento ${ }^{11}$.

\section{Intervenção de enfermagem em caso de cardioversão elétrica}

Antes do procedimento, deve ser feito exame de sangue para controle dos eletrólitos e devem ser corrigidos antes da cardioversão. Para a cardioversão elétrica, o paciente precisa ficar em jejum de 8 horas. 0 paciente necessita de um acesso venoso, controle da pressão arterial, monitorização do eletrocardiograma e oxímetro de pulso ${ }^{13}$. Considerando a cardioversão elétrica como um procedimento doloroso, o paciente precisa ser bem sedado ou anestesiado. Existe sempre o risco de indução de fibrilação ventricular com um choque elétrico e por isso ele deve ser sempre sincronizado com o complexo QRS e o procedimento feito em um ambiente que tenha 0 equipamento de emergência para intubação e possa assistir uma parada cardíaca.

\section{Intervenção de enfermagem em caso de Ablação por radiofreqüência}

É necessário um jejum de 8 horas para a realização da ablação por radiofreqüência. 0 paciente deve ser devidamente sedado para ser submetido ao procedimento, a pressão arterial, eletrocardiograma e oxímetro de pulso são monotorizados e um acesso venoso é obtido. 0 desfibrilador deve estar disponivel e pronto para utilização imediata. Devido à heparinazação, deve-se obter níveis de TCA (Tempo de Coagulação Ativado), que tem de ser aferido a cada 30 min. Durante o procedimento, a enfermagem deve estar sempre atenta para possíveis complicações como tamponamento cardíaco, tromboembolia e arritmias. Após o procedimento, os locais de inserção de cateteres de ablação devem ser cuidadosamente monitorizados para evitar possível sangramento e formação de hematoma. 0 paciente deve ficar acamado por 6 a 8 horas e não deve dobrar a perna para evitar sangramento no local de inserção dos cateteres ${ }^{14}$. Os sinais vitais e saturação de oxigênio devem permanecer monitorizados após o procedimento. 0 paciente geralmente recebe alta em um a dois dias após o procedimento. 


\section{CONCLUSÃO}

A fibrilação atrial é um problema de saúde crescente. Como a população mundial envelhece progressivamente, conseqüentemente o número de pacientes que têm fibrilação atrial apresenta um aumento progressivo da sua ocorrência.

\section{Referências}

1.Furberg $C D$. Prevalence of atrial fibrillation in elderly subjects: the cardiovascular health study. Am J Cardiol 1994; 74:236-41.

2.Le Heuzy JYI. Cost of care distribution in atrial fibrillation patients: the COCAF study. Am Heart J 2004;147(1): 121-26.

3.Cruz FES. Reversibility of tachycardia-induced cardiomyoopathy after cure of incessant supraventricular tachycardia. J Am Coll Cardiol $1990 ; 16: 739-44$.

4. Sociedade Brasileira de Cardiologia. Diretrizes de fibrilação atrial. Arq Bras Cardiol 2003; 81 ( supl 6): 1-24

5.Wyse DG. For the Atrial Fibrillation Follow-up Investigation of Rhythm Management(AFFIRM) investigators: a comparison of rate control and rhythm control in patients with atrial fibrillation. N Engl J Med 2002: 347(23): 1825-33.

6. Villani GQ. Anticoagulation in atrial fibrillation: what is certain and what is to come? Eur Heart J 2003;5(supl h): h45-h50.

7.Ricard PH. Cardioversion of atrial fibrillation: how and when? Eur Heart J 2003; 5(supl h): h 40- h 44.

8. Santoro DC. Situação do sistema de saúde no Brasil e os cuidados desenvolvidos nas unidades de terapia intensiva. Esc Anna Nery Rev Enf 2001 ago; 5(2): 259-61

9. Jais $P$. Radiofrequency ablation for atrial fibrillation. Eur Heart J 2003, 5(supl h): h 34- h 39.

10. Sociedade Brasileira de Cardiologia.Diretrizes para avaliação e tratamento de pacientes com arritmias cardíacas. Arq Bras Cardiol 2002; 79( sup 5): $1-50$

11.Santoro DC. 0 cuidado de enfermagem na unidade coronariana: um ensaio sobre a dimensão da subjetividade no cuidar. [tese de doutorado] Rio de Janeiro(RJ): Escola de Enfermagem Anna Nery /UFRJ; 2000.

12. Viana CO, Optiz SP, Linhares JC, Cassiani SHDB, Miasso AJ. Segurança do paciente hospitalizado: avaliação do grau de conhecimento sobre a terapia medicamentosa. Esc Anna Nery R Enferm 2004 ago; 8(2): 235-42.

13. Santoro DC, Oliveira CM. Conduta da equipe de enfermagem diante das alterações clínicas com síndrome coronariana. Esc Anna Nery Rev Enferm 2004 ago; 8(2): 267-74

14.Vanheusden LMS. Participação e cuidados da enfermagem em procedimentos de ablação com radiofrequência nas taquicardias supraventriculares. Arq Bras Cardiol 1994; 62 (5): 361-67.
A educação, acompanhamento e cuidado de enfermagem são primordiais para os pacientes portadores de fibrilação atrial. A contribuição do profissional de enfermagem dentro do conceito multidisciplinar amplia o sucesso na condução dos pacientes proporcionando um desfecho favorável no período pré, intra e pós-hospitalar.

\section{Sobre as Autoras}

\section{Lutgarde Magda Suzanne Vanheusden}

Escola de enfermagem Anna Nery - Rio de Janeiro.

\section{Deyse Conceição Santoro}

Escola de enfermagem Anna Nery - Rio de Janeiro. e-mail: fcruz@ alternex.com.br 